\title{
A Sensitive Line Search in Circumstellar Fnvelopes
}

\author{
Nguyen-Q-Rieu ${ }^{1}$, S. Deguchi, H. Izumiura \\ N. Kaifu, M. Ohishi, H. Suzuki, and N. Ukita
}

Nobeyama Radio Observatory, Minamimaki, Minamisaku, Nagano 384-13, JAPAN

1. Present Address, Observatoire de Paris-Meudon

Abstract A molecular 1 ine search in the range between 85 and $89 \mathrm{GHz}$ has been performed in the circumstellar envelopes of 11 evolved stars. Emissions of ${ }^{29} \mathrm{SiO} J=2-1,{ }^{28} \mathrm{SiO} J=2-1, \operatorname{HCN} \mathrm{J}=1-0$,

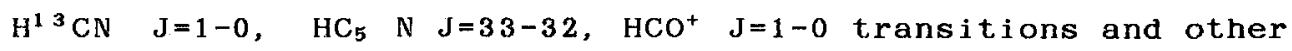
transitions of $\mathrm{C}_{2} \mathrm{H}, \mathrm{C}_{4} \mathrm{H}$, and $\mathrm{C}_{3} \mathrm{~N}$ have been observed in 11 stars. We have detected the ground state ${ }^{29} \mathrm{SiO} J=2-1$ maser in several stars. We have also detected HCN emission in VY CMa. A narrow $\mathrm{H}^{13} \mathrm{CN}$ spike feature near the central velocjty has been found in the spectrum of CRL 2688 .

Introduction An increasing number of molecular transitions have been detected in the circumstellar envelopes of evolved objects through recent sensitive searches with large radio telescope. Some progress has been made toward the understanding of chemical processes at work in the circumstellar medium, based on observational results and no-LTE chemical calculations. Using the VLA and Hat Creek interferometers, Nguyen-Q-Rieu et al. (1987) and Bieging and Nguyen-Q-Rieu (1988) found that, the $\mathrm{NH}_{3}$ and $\mathrm{HCN}$ shells of CRL 2688 are troid while $\mathrm{HC}_{7} \mathrm{~N}$ is distributed in a spheroidal halo. The molecule HCN which was believed to exist only in carbon-rich atmosphere has been found in oxygen-rich envelopes (Deguchi and Goldsmith 1985). Cyanopolyyne and hydrocarbon have been detected in carbon stars (Saito et al.1987). Above considerations suggest that the envelope of cool stars is rich in physical phenomena and prompt us to perform a search for molecular emission in stellar envelopes. 

and May 1987 using the $45 \mathrm{~m}$ radio telescope at Nobeyama. We search for molecular transitions in the range between 85.00 and $89.25 \mathrm{GHz}$ in sample of 11 envelopes known to be $\mathrm{rich}$ in carbon and oxygen. An acousto-optical spectrometer (AOS) with 8 arrays and 2048 channels each was used. We have detected many 1 ines in a sample of 11 stars. The HCN molecules have been discoverd in VY CMa, an oxygen-rich star, in which carbon was supposed to be bound as $\mathrm{CO}$ and $\mathrm{HCN}$ was not expected. The abundance of $\mathrm{HCN}$ in the envelope of VY CMa is calculated to be $6 \times 10^{-9}$ per H2. We have found a spike feature in the $\mathrm{H}^{13} \mathrm{CN}$ spectrum in CRL 2688 . This source, a bipolar reflection nebula known as Egg Nebula, has been observed at infrared and radio frequencies. The origin of the spike feature at $29 \mathrm{Km} \mathrm{s}^{-1}$ in the $\mathrm{HCN}$ spectrum is not clear and no counterpart appears in the $\mathrm{H}^{12} \mathrm{CN}$ spectrum. Plausible is that it is an anomalous excitation.

\section{References}

Bieging, J. H., and Nguyen-Q-Rieu, 1988, Ap. J. (in press)

Deguchi, S., and Goldsmith, P. F., Nature 317, 336

Nguyen-Q-Rieu et al., 1986, A. Ap. 165, 204

Saito, S. et al., 1987, Publ. A. S. Japan 39, 193
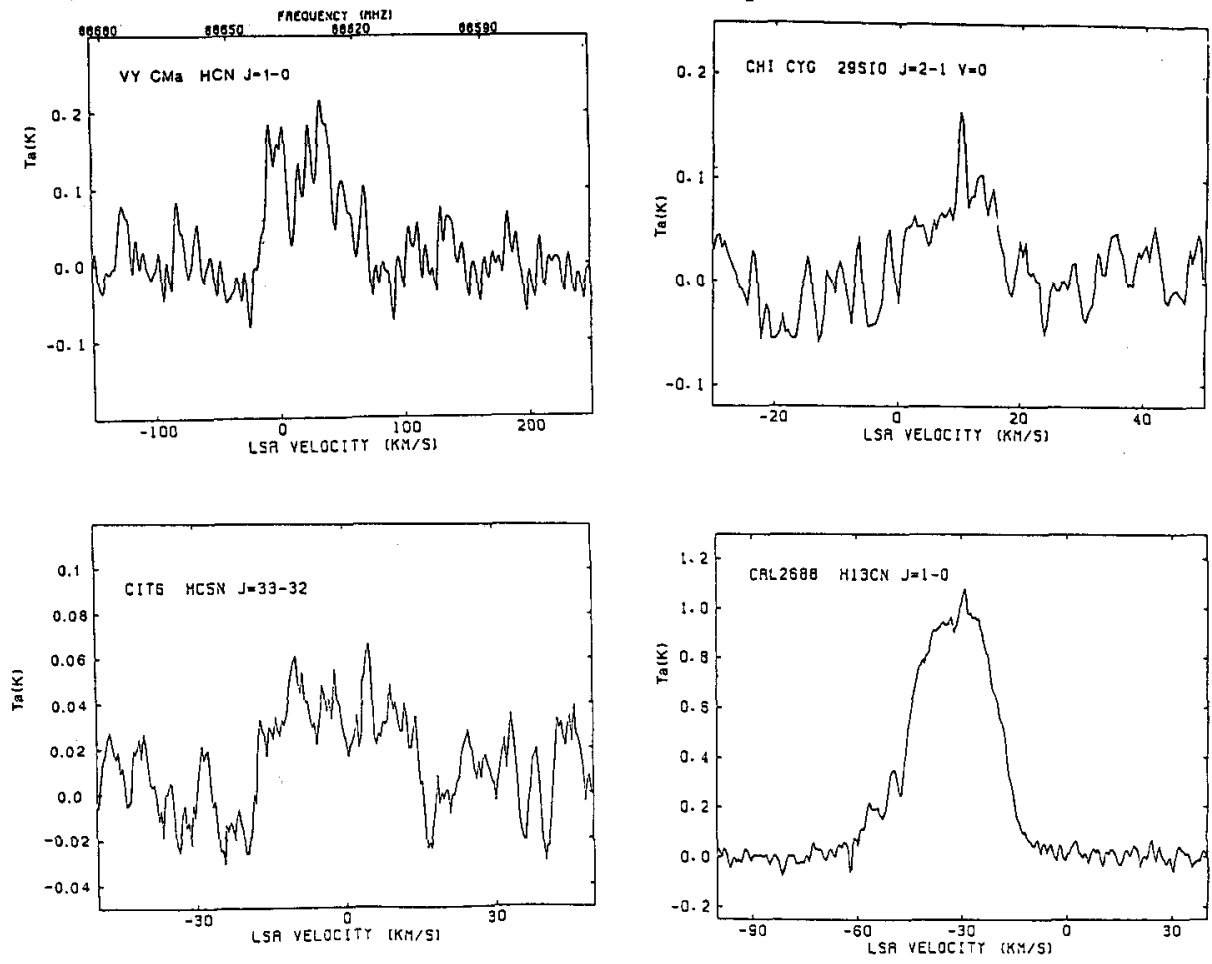